\title{
Comparative transcriptomics of two environmentally relevant cyanobacteria reveals unexpected transcriptome diversity
}

\author{
Karsten Voigt ${ }^{1}$, Cynthia M Sharma ${ }^{2}$, Jan Mitschke ${ }^{1}$, S Joke Lambrecht ${ }^{1}$, Björn Voß ${ }^{1}$, \\ Wolfgang R Hess ${ }^{1}$ and Claudia Steglich ${ }^{1}$ \\ ${ }^{1}$ Faculty of Biology, University of Freiburg, Freiburg, Germany and ${ }^{2}$ Research Center for Infectious \\ Diseases (ZINF), University of Würzburg, Würzburg, Germany
}

\begin{abstract}
Prochlorococcus is a genus of abundant and ecologically important marine cyanobacteria. Here, we present a comprehensive comparison of the structure and composition of the transcriptomes of two Prochlorococcus strains, which, despite their similarities, have adapted their gene pool to specific environmental constraints. We present genome-wide maps of transcriptional start sites (TSS) for both organisms, which are representatives of the two most diverse clades within the two major ecotypes adapted to high- and low-light conditions, respectively. Our data suggest antisense transcription for three-quarters of all genes, which is substantially more than that observed in other bacteria. We discovered hundreds of TSS within genes, most notably within 16 of the 29 prochlorosin genes, in strain MIT9313. A direct comparison revealed very little conservation in the location of TSS and the nature of non-coding transcripts between both strains. We detected extremely short $5^{\prime}$ untranslated regions with a median length of only 27 and 29 nt for MED4 and MIT9313, respectively, and for $\mathbf{8 \%}$ of all protein-coding genes the median distance to the start codon is only $10 \mathrm{nt}$ or even shorter. These findings and the absence of an obvious Shine-Dalgarno motif suggest that leaderless translation and ribosomal protein S1-dependent translation constitute alternative mechanisms for translation initiation in Prochlorococcus. We conclude that genomewide antisense transcription is a major component of the transcriptional output from these relatively small genomes and that a hitherto unrecognized high degree of complexity and variability of gene expression exists in their transcriptional architecture.

The ISME Journal (2014) 8, 2056-2068; doi:10.1038/ismej.2014.57; published online 17 April 2014

Subject Category: Integrated genomics and post-genomics approaches in microbial ecology

Keywords: antisense RNA; cyanobacteria; dRNA-seq; Prochlorococcus; transcriptomics; prochlorosin
\end{abstract}

\section{Introduction}

Prochlorococcus is a marine and unicellular cyanobacterium that populates the oligotrophic open oceans between $40^{\circ} \mathrm{N}$ and $40^{\circ} \mathrm{S}$ (Partensky et al., 1999). In these areas, Prochlorococcus numerically dominates the phytoplankton with up to $5 \times 10^{5}$ cells per $\mathrm{ml}$, contributing a significant fraction of the photosynthetic biomass (Goericke and Welschmeyer, 1993; Vaulot et al., 1995). The oceanic ecosystem is strongly affected by the interplay between Prochlorococcus, cyanophage that infect Prochlorococcus and alphaproteobacteria of the SAR11 clade. Whereas the latter is dependent on organic matter produced by primary producers such as Prochlorococcus, cyanophage contribute to this

Correspondence: C Steglich, Faculty of Biology, University of Freiburg, Freiburg D-79104, Germany.

E-mail: claudia.steglich@biologie.uni-freiburg.de

Received 10 December 2013; revised 3 March 2014; accepted 5 March 2014; published online 17 April 2014 interaction by lysing Prochlorococcus, hence making the organic matter available (Thompson et al., 2013). Two distinct ecotypes have been defined according to their adaptation to high-light (HL; for example, strain MED4) or low-light regimes (LL; for example, strain MIT9313) (Moore et al., 1995). However, other environmental factors, such as temperature, nutrients and competitor abundance, also affect the distribution of ecotypes (Johnson et al., 2006). The HL and LL ecotypes can be further divided into six distinct subclades, two within the high-light ecotype (HLI and HLII) and four within the low-light ecotype (LLI-LLIV) (Kettler et al., 2007). Within this classification, MED4 and MIT9313 represent the two most distantly related Prochlorococcus clades (HLI vs LLIV). Although their $16 \mathrm{~S}$ rRNA is $97.5 \%$ identical, their proteincoding potential differs, with 1955 annotated protein-coding genes for MED4, 2843 for MIT9313 and a shared number of only 1447 genes (as determined by BLAST search using an e-value of $10^{-8}$ ). The suite of strain-specific open reading 
frames (ORFs) includes genes encoding photosynthetic proteins and also genes that are involved in nutrient uptake, assimilation and metabolic functions (Rocap et al., 2003; Kettler et al., 2007). This suggests that there could be ecotype-specific regulatory elements that specifically control these functions and therefore can be expected to differ between the two strains.

The genomes of 12 isolates of Prochlorococcus have been completely sequenced, revealing a trend toward compact and streamlined genomes (Dufresne et al., 2003; Rocap et al., 2003; Kettler et al., 2007). Such genomic streamlining has been recognized to be important in many other open ocean marine bacteria, such as representatives of the SAR11 clade of alphaproteobacteria (Grote et al., 2012), and has been interpreted as a typical adaptation of the marine bacterioplankton to oligotrophy (Swan et al., 2013). Due to this streamlining, Prochlorococcus genomes are depleted in guanine and cytosine residues, are densely packed with small intergenic regions, and contain only a few genes encoding proteins involved in transcription, signal transduction and the regulation of gene expression (Dufresne et al., 2003; Rocap et al., 2003; Kettler et al., 2007). Such genomic features have also been recognized for many other bacterioplankton species (Swan et al., 2013).

Despite their densely packed genomes and relatively low number of transcriptional protein regulators (only five- and eight-sigma factors, six and seven response regulators as well as six and 10 histidine kinases in MED4 and MIT9313, respectively (Scanlan et al., 2009)), Prochlorococcus is capable of adapting to environmental perturbations. The identification of relatively high numbers of non-coding RNAs (ncRNAs) in MED4 (17 small RNAs and 24 antisense RNAs (asRNAs)) suggested that regulatory RNAs may play an important role in the regulation of gene expression in Prochlorococcus (Steglich et al., 2008). For the ncRNA Yfr1, a function in the control of major outer-membrane proteins PMM1119 and PMM121 was shown (Richter et al., 2010). More recently, antisense transcripts were detected for $73 \%$ of all genes in MED4 at some point in time over the diel cycle (Waldbauer et al., 2012). This percentage is substantially higher than the previously reported fraction of genes associated with asRNA molecules in any bacterium, which was at $46 \%$ the highest in Helicobacter pylori and less in other bacteria (Georg et al., 2009). Moreover, the abundance of asRNAs was reported to be relatively high in MED4 with an average of $35 \%$ of the corresponding mRNA concentration (Waldbauer et al., 2012). However, despite the wealth of available genome information, insight into the transcriptional architecture and the numbers and types of potentially regulatory RNA molecules remains largely fragmentary and limited to MED4.

On the basis of high-density microarray hybridizations, several studies have provided valuable transcriptomic information on genes of MED4 and to some extent on genes of MIT9313 that are differentially expressed under environmentally relevant perturbations such as light stress, phosphorus, nitrogen and iron starvation and phage infection (Martiny et al., 2006; Steglich et al., 2006; Tolonen et al., 2006; Lindell et al., 2007; Thompson et al., 2011) and hence are involved in the adaptation to those conditions. However, except for the experimental determination of 25 transcriptional start sites (TSS) in MED4 (Vogel et al., 2003) and the mapping of the TSS of $c p e B$ (Steglich et al., 2005), psbA, $p s b C$, psbD and pcbA genes (Garczarek et al., 2001), there is no information on promoters that are actually utilized in Prochlorococcus. The combination of our study with previous microarray analyses will promote the targeted search for new regulatory promoter elements in Prochlorococcus, which so far is restricted on the knowledge of the NtcA regulon (Tolonen et al., 2006) and computational modeling studies of the cyanobacterial Pho, LexA and cAMP receptor protein regulons (Su et al., 2007; Li et al., 2010; $\mathrm{Xu}$ and $\mathrm{Su}, 2009$ ).

Here, we present a comparative analysis of the transcriptomes of two Prochlorococcus strains representing the two most diverse clades within the two major ecotypes adapted to high- and lowlight conditions, respectively. We used three different high-throughput methodologies (454 and Solexa sequencing platforms) in combination with a differential RNA-seq approach selective for the analysis of primary transcriptomes and global mapping of TSS (Sharma et al., 2010), as well as Affymetrix highdensity microarrays for the independent verification of antisense transcripts. We complemented these with computational and experimental validation of the selected genes. We present genome-wide TSS maps for both strains at a single nucleotide resolution that enables the interrogation of transcriptional activity in a comparative fashion. We found a high degree of antisense transcription and identified new ncRNAs. With the exception of photosynthesisrelated genes, the TSS of protein-coding genes and asRNAs are not conserved, suggesting a high degree of variability in their transcriptional architecture.

\section{Materials and methods}

Culture growth conditions, RNA isolation and northern blot analysis

Cells were grown at $22^{\circ} \mathrm{C}$ in AMP1 medium (Moore et al., 2007) under $10-30 \mu \mathrm{mol}$ quanta $\mathrm{m}^{-2} \mathrm{~s}^{-1}$ continuous white cool light and harvested in an exponential growth phase. For microarray analysis and northern verifications, the cells were subjected to several stress conditions for $30 \mathrm{~min}$ : light stress (light shifts from 10 to $100 \mu \mathrm{E}$ or darkness, respectively, or from 30 to $300 \mu \mathrm{E}$ ) and temperature stress (shifts from 22 to 12 or $32{ }^{\circ} \mathrm{C}$, respectively). For nitrogen and iron starvation, the cells were washed twice in nitrogen- or iron-free medium and grown in 
minus $\mathrm{N}$ or minus $\mathrm{Fe}$ medium for 2,3 or 6 days. Alternatively, iron depletion was induced via the addition of $0.6 \mu \mathrm{M}$ DFB for $24-48 \mathrm{~h}$. Total RNA was extracted from the cells via filtration following the hot phenol method (Steglich et al., 2006) or a modified protocol using PGTX buffer, which consists of $4.2 \mathrm{M}$ phenol amended with $6.9 \%$ v/v glycerol, $5 \mathrm{~mm}$ 8-hydroxyquinoline, $15.6 \mathrm{~mm}$ Na-EDTA, $0.1 \mathrm{M}$ sodium acetate, $0.8 \mathrm{M}$ guanidine thiocyanate and $0.48 \mathrm{M}$ guanidine hydrochloride (Pinto et al., 2009). The MIT9313 cells were lysed prior to RNA extraction using a cell disruption device (Precellys, PeqLab, Erlangen, Germany) applying 6 cycles at power level of 6.5 for $20 \mathrm{~s}$. Northern hybridizations were performed as described (Stazic et al., 2011).

454 and Solexa dRNA sequencing and computational analysis

Details for the 454 differential RNA-seq approach protocol are described in Sharma et al. (2010) and Mitschke et al. (2011). For both strains, a (+) cDNA library synthesized from a primary RNA pool of exponentially grown cells (total RNA treated with Terminator $5^{\prime}$ P-dependent exonuclease, Epicenter, San Diego, CA, USA) and a (-) cDNA library synthesized from total RNA were generated. To produce the RNA 5'-monophosphates necessary for RNA linker ligation, the samples were treated with tobacco acid pyrophosphatase (after exonuclease treatment if applied). Each RNA sample was ligated to an RNA oligonucleotide containing a unique sequence tag (see Supplementary Table S1). cDNA libraries were sequenced on a Roche FLX sequencer (Basel, Switzerland) and the resulting data were analyzed as described (Sittka et al., 2008). For MED4 (NCBI accession number BX548174.1), a total of 60457 and 61633 sequence reads and for MIT9313 (NCBI accession number BX548175.1) a total of 104037 and 113994 sequence reads were obtained for the $(-)$ and $(+)$ libraries, respectively. From these, 47339 and 46555 sequence reads for MED4 as well as 72988 and 74389 sequence reads for MIT913 were $\geqslant 18 \mathrm{nt}$ in length. After filtering out ribosomal RNA matches, 40291 and 40739 reads remained in the MED $(-)$ and $(+)$ libraries, respectively, and 53287 and 59761 reads remained in the MIT9313 $(-)$ and $(+)$ libraries, respectively.

TSS were determined from $(+)$ libraries following the same work flow as described in Mitschke et al. (2011). An individual position-specific scoring matrix for the -10 element of MED4 or MIT9313 was derived, taking the putative TSS of all expressed protein-coding genes of the respective transcriptome into account (Supplementary Tables S2 and S3). Subsequently, a minimum - 10 element score of 2.0 followed by at least two sequences was set for the TSS minimum threshold. TSS were classified into four groups: gTSS (start sites of annotated proteincoding genes within a range of 0 to $100 \mathrm{nt}$ upstream of the ORF), iTSS (start sites within annotated genes), aTSS (start sites opposite to annotated genes or within $30 \mathrm{nt}$ of its $5^{\prime}$ and $3^{\prime}$ untranslated regions (UTR) giving rise to asRNAs) or nTSS (all remaining TSS giving rise to potential ncRNAs) (Supplementary Tables S4 and S5). For classification, gTSS were prioritized over aTSS and iTSS.

cDNA libraries for Solexa sequencing were prepared in the same manner as for 454 sequencing with one modification: the RNA for the ( - ) cDNA library preparation was neither treated with terminatordependent exonuclease nor treated with tobacco acid pyrophosphatase, resulting in a $(-)$ cDNA library entirely depleted of the primary RNA pool (because triphosphate $5^{\prime}$-termini cannot be ligated to the RNA linker oligonucleotide). For cDNA synthesis and the amplification, Solexa-specific TrueSeq sequencing primers with a unique sequence tag for each library were used. The cDNA libraries were analyzed on an Illumina GA IIx sequencer (San Diego, CA, USA). Sequence data have been deposited in NCBI's Sequence Read Archive under accession number SRR1045147 for MED4 and SRR1045146 for MIT9313. Sequence lengths of $72 \mathrm{nt}$ were obtained for both MED4 libraries and for the (-) MIT9313 library, and sequence lengths of 26 nt were obtained for the $(+)$ MIT9313 library. For MED4 totals of 8278893 and 6324321 sequence reads, and for MIT9313 totals of 10689054 and 8245154 sequence reads, were obtained for the $(-)$ and $(+)$ libraries, respectively. The sequences were mapped to the respective genome with segemehl (Hoffmann et al., 2009), resulting in 7429557 and 5750991 redundant read mappings for MED4 and 14945219 and 4663103 redundant read mappings for MIT9313. After filtering out ribosomal RNA matches, 6389217 and 6389217 reads remained in the MED4 $(-)$ and $(+)$ libraries and 4251797 and 3423681 reads remained in the MIT9313 $(-)$ and $(+)$ libraries. The $(+)$ libraries of MED4 and MIT9313 were normalized to a read value per million bp and million Solexa reads. A minimum -10 element score of 2.0 followed by at least 27 reads for MED4 and 25 reads for MIT9313 starting within a window of five to seven nucleotides (three sequence reads each after normalization) were set as minimum thresholds for the definition of a TSS. The same criteria as above for the 454 data sets were applied to classify TSS into gTSS, iTSS, aTSS or nTSS. TSS with the same -10 element were clustered to one entry. The entries were subsequently clustered when - 10 elements were located within a 6-nt window (Supplementary Tables S4 and S5).

\section{Prediction of transcriptional units}

Transcriptional units are genomic segments with uniform read coverage from the $(-)$ cDNA library. Segmentation of the data into transcriptional units with RNASeg (to be published elsewhere) was performed based on the $(-)$ cDNA library and the 
previously defined gTSS, aTSS and nTSS. For this, primary read starts were normalized to 1 for starts and to 0 for non-starts to assure that only previously defined TSS were used. A maximum segment length of $7000 \mathrm{nt}$ and $10000 \mathrm{nt}$ was defined for MED4 and MIT9313, respectively. If the distance between two subsequent TSS exceeded this length, this distance was used for the corresponding genomic region. The minimum segment length was set to 45 for both strains to cover small transcripts. For transcript segments, the maximum distance to a TSS was set to 10 for both strains and the mean secondary read coverage was set to 1 for MED4 and to 0.5 for MIT9313. Segments with lower coverage were defined as non-transcripts. If the transcript segment exceeded $70 \%$ coverage of an already classified region, the segment was assigned to the appropriate class.

\section{Determination of conserved TSS and functional enrichment \\ TSS were considered as conserved between MED4 and MIT9313 if their distance from the respective start codon differed no more than $10 \mathrm{nt}$ (gTSS) or if their relative distance with respect to a protein alignment was not more than $10 \mathrm{nt}$ (iTSS and aTSS). \\ Conserved TSS between MED4 and MIT9313 of each TSS class were inspected for functional enrichment using the DAVID web interface (http:// david.abcc.ncifcrf.gov/). Functional classes with an enrichment score above 1.3, corresponding to a $P$-value below 0.05 , were considered as true enriched functional categories.}

Microarray labeling, hybridization, normalization and segmentation

For the detection of asRNA expression signals on microarrays, RNA was first treated with TURBO DNase (Life Technologies, Carlsbad, CA, USA). In total, 6 units of DNase were added to the RNA samples and digestion of DNA was carried out in three consecutive incubation steps, each at $37^{\circ} \mathrm{C}$ for $10 \mathrm{~min}$. RNA was either directly labeled (without cDNA synthesis) with the Kreatech (Amsterdam, The Netherlands) 'ULS labeling kit for Affymetrix arrays' with Cy3 according to the manufacturer's protocol or previously depleted of rRNA using Ambion's (Carlsbad, CA, USA) MICROBExpress kit. Of note, probes for the Affymetrix gene expression arrays were designed for hybridization with cDNA. Therefore, direct hybridization with RNA results in signals for the respective asRNA strand. Fragmentation was performed at $70^{\circ} \mathrm{C}$ for $15 \mathrm{~min}$ using fragmentation buffer (Ambion). Hybridization and scanning were performed according to Affymetrix protocols for E. coli (http:// www.affymetrix.com/support/technical/manual/expression_manual.affx and Steglich et al., 2006) using $2.5 \mu \mathrm{g}$ of total RNA (or $1 \mu \mathrm{g}$ rRNA depleted RNA) on an Affymetrix high density array MD4-9313, which contains probes for Prochlorococcus MED4 and MIT9313. The custom array covers all gene-coding regions with a probe pair (match and mismatch) every 80 bases and every 45 bases in the intergenic regions in both the sense and antisense orientations. For both strains, one microarray each was hybridized with RNA extracted from cells grown under standard conditions. A second microarray was hybridized with pooled RNA of cells subjected to different stress conditions (light and temperature stress, nitrogen and iron starvation). Microarray data have been deposited in NCBI's Gene Expression Omnibus under accession number GSE17075. Microarray expression data of single probes were quantile-normalized using the $\mathrm{R}$ package LIMMA (Smyth, 2005). The expression threshold value was individually evaluated for MED4 and MIT9313 and was set to 250 and 400, respectively. Consecutively expressed probes (in at least one condition, stress or standard) were combined into segments allowing one probe below the threshold within the segment if the following probe showed expression above the set threshold.

\section{Verification of potential ncRNAs via secondary} structure prediction using RANDfold

For the computational prediction of ncRNAs, we extracted candidate sequences based on nTSS reads and analyzed their thermodynamic structural stability with RANDfold (Bonnet et al., 2004). We used two different approaches to identify promising ncRNA candidates: a sliding window approach and an expanding window approach. In the expanding window approach, all possible lengths between 30 and 200 nt were folded one by one; in the sliding approach, windows of $100 \mathrm{nt}$ beginning at the start of the predicted nTSS were shuffled in a range of 0-300 nt in 10-nt increments. For both approaches, $P$-values below 0.05 were counted and summarized in a hit list (Supplementary Tables S9 and S10).

\section{Results and discussion}

The primary transcriptomes of two Prochlorococcus strains are highly diverse

To characterize the transcriptomes of the two Prochlorococcus strains, total RNA samples of MED4 and MIT9313 cultures grown under standard conditions were used to generate two strandspecific cDNA libraries that allow for a differential sequencing of primary transcripts and processed transcripts. In bacteria, most primary transcripts carry a triphosphate at their $5^{\prime}$ ends resulting from initiation of transcription, whereas processed or degraded RNA fragments possess a $5^{\prime}$ mono-phosphate or $5^{\prime}$ hydroxyl. These differences were employed here by synthesizing two cDNA libraries for each strain: one from the original, untreated RNA pool containing both primary and processed transcripts ( - - cDNA library), and one from RNA 
that was enriched in primary transcripts by selective degradation of RNAs containing mono-phosphates using Terminator $5^{\prime}$ P-dependent exonuclease $((+)$ cDNA library) (Sharma et al., 2010; Mitschke et al., 2011). After sequencing of these differential libraries of both strains, reads were mapped to the genomes of MED4 and MIT9313 (Table 1), and two separate position-specific scoring matrixes (Supplementary Tables S2 and S3) were generated for the -10 elements of both strains. These position-specific scoring matrixes were based on the nucleotides at positions -7 to -12 of all mapped gTSS with a minimum of 11 reads (9927 $5^{\prime}$ ends for MED4 and 13626 for MIT9313). Subsequently, a positionspecific scoring matrix minimum score of 2.0 for the -10 element served as a filter criterion for the identification of TSS. For the same sequence motif of the -10 element, different score values were obtained for MED4 and MIT9313 due to the difference in GC content $(30.8 \%$ for the MED4 genome and $47.5 \%$ for MIT9313). Of the 26 previously characterized gTSS (TSS of proteincoding genes) (Vogel et al., 2003; Steglich et al., 2005), 18 were identified directly. Two of the eight missing gTSS had a score below 2.0 for the new -10 element, and four failed the read number criterion, indicating that there is an even higher number of active TSS in MED4. Consistent with previous results (Vogel et al., 2003), the targeted search for the -35 promoter element 'TTGACA' yielded a very small subset of $3.3 \%$ (MED4) or $2.1 \%$ (MIT9313) TSS with a conserved E. coli-like - 35 element.

In total, 4126 and 8587 TSS were defined for MED4 and MIT9313, resulting from Solexa and 454 sequencing data that were further classified into gTSS (protein-coding genes), aTSS (asRNAs), nTSS (potential ncRNAs) or iTSS (within genes) (for more details, see Material and methods) (Supplementary Tables S4 and S5 and Supplementary Files S1-S6). For approximately half of all protein-coding genes (MED4, 49\% and MIT9313, 41\%), a gTSS could be assigned. Surprisingly, there was no good correlation between highly expressed genes in MED4 and those in MIT9313 (Supplementary Table S6). Highly expressed TSS are dominated by gTSS, followed by nTSS, including transfer-messenger RNA - the only nTSS shared between both strains among the top 20-and the newly verified ncRNAs Yfr23 (MED4), Yfr102 and Yfr103 (MIT9313) (Supplementary Table S6). For more details, see the section on ncRNAs. The only gTSS in the top 20 list that occurs in both strains is driving transcription of the psbEFLJ operon, encoding the cytochrome b559 alpha and beta subunits and the $\mathrm{L}$ and $\mathrm{J}$ proteins of PSII (Supplementary Table S6). Other examples of the top 20 list provide a clear connection of gene expression to the physiology of Prochlorococcus for example, as illustrated by the gTSS for genes such as $p c b$ or hli10 in MED4, which encode the single light harvesting protein and one of the high-light-inducible proteins. Another difference between both transcriptomes is the density of TSS. The median distance between two consecutive TSS is $353 \mathrm{nt}$ and $563 \mathrm{nt}$ for MIT9313 and MED4, respectively. The functional relevance of the higher TSS density in MIT9313 remains unclear.

\section{Conservation of distinct TSS of photosynthesis-related genes}

We searched for gTSS conserved between both strains (defined by an identical distance to the translational start site $\pm 10 \mathrm{nt}$ ). Of the 1447 MED4MIT9313 shared protein-coding genes, 436 were

Table 1 Summary of genome and transcriptome information. Sequencing numbers are given for primary libraries only

\begin{tabular}{|c|c|c|c|c|c|}
\hline & \multicolumn{2}{|c|}{ MED4 } & \multicolumn{2}{|c|}{ MIT9313 } & Reference \\
\hline $\begin{array}{l}\text { Ecotype adapted to } \\
\text { Hfq } \\
\text { Genome size } \\
\text { Annotated protein-coding genes }\end{array}$ & \multicolumn{2}{|c|}{$\begin{array}{l}\text { High light } \\
\text { No } \\
1.66 \mathrm{Mbp} \\
1974\end{array}$} & \multicolumn{2}{|c|}{$\begin{array}{l}\text { Low light } \\
\text { Yes } \\
2.41 \mathrm{Mbp} \\
2.894\end{array}$} & \multirow[t]{2}{*}{$\begin{array}{l}\text { Moore et al., } 1995 \\
\text { Axmann et al., } 2005 \\
\text { Rocap et al., } 2003 \\
\text { Kettler et al., } 2007\end{array}$} \\
\hline Annotated protein-coding genes & 454 sequencing & Solexa sequencing & 454 sequencing & Solexa sequencing & \\
\hline Total number of mapped reads & 61633 & 5750991 & 113994 & 4663103 & \multirow{11}{*}{ This work } \\
\hline Number of reads w/o ribosome & 40739 & 5425895 & 59761 & 3712862 & \\
\hline Total number of TSS ${ }^{\mathrm{a}}$ & 1643 & 3459 & 3402 & 7410 & \\
\hline gTSS & 608 & 1059 & 801 & 1284 & \\
\hline iTSS & 423 & 658 & 928 & 2256 & \\
\hline aTSS & 520 & 1566 & 1372 & 3231 & \\
\hline nTSS & 92 & 176 & 300 & 639 & \\
\hline aTSS with most reads & $\begin{array}{c}\text { asPMED4_06291 } \\
(c l p B, 52 \text { reads })\end{array}$ & $\begin{array}{c}\text { asPMED4_04671 } \\
\text { (cons. hyp., } 21506 \text { reads) }\end{array}$ & $\begin{array}{l}\text { asP9313_16021 } \\
(\text { priA, } 184 \text { reads })\end{array}$ & $\begin{array}{c}\text { asP9313_06941 } \\
\text { (recG, } 3542 \text { reads) }\end{array}$ & \\
\hline gTSS with most reads & $\begin{array}{c}\text { PMED4_11782 } \\
\text { (cons. hyp., } 482 \text { reads) }\end{array}$ & $\begin{array}{c}\text { PMED4_16461 } \\
\text { (groES, } 93670 \text { reads) }\end{array}$ & $\begin{array}{c}\text { P9313_14931 } \\
\text { (psbD, 263 reads) }\end{array}$ & $\begin{array}{c}\text { P9313_20011 } \\
(\text { smc }, 124649 \text { reads })\end{array}$ & \\
\hline nTSS with most reads & Yfr11 (1700 reads) & Yfr16 (1 184303 reads) & Yfr103 (413 reads) & Yfr2-5 (48 227 reads) & \\
\hline Filter criteria for TSS mapping & $\geqslant 2$ reads, score $\geqslant 2.0$ & $\geqslant 26$ reads, score $\geqslant 2.0$ & $\geqslant 2$ reads, score $\geqslant 2.0$ & $\geqslant 24$ reads, score $\geqslant 2.0$ & \\
\hline
\end{tabular}

Abbreviation: w/o, without.

${ }^{a}$ Total number of TSS after clustering of neighboring TSS that start $\pm 10 \mathrm{nt}$ of each other or have a similar $\pm 3 \mathrm{nt}-10$ element (as described above) and removal of potential processing sites (primary coverage is lower than secondary coverage). 
associated with one or more gTSS. From these, 214 possess a conserved gTSS (Supplementary Table S7). A functional enrichment analysis revealed that photosynthesis-related genes were highly overrepresented in this group (enrichment score 7.54). Among the photosynthesis-related genes, those encoding proteins of both photosystem I ( $p s a C, p s a D$, psaE and $p s a F$ ) and photosystem II (psbA, psbB, psbE, $p s b H, p s b O$ and psb28) are found. Other genes in this category encode electron transfer proteins (petE, petH) and cytochrome b6/f complex components $($ petB). As many of these genes are located in operons, an even higher number of photosynthesisrelated genes belong to this class, illustrated by the conserved gTSS upstream of $p s b E$, in fact driving the transcription of psbEFLJ. For other functional classes, the enrichment was not as pronounced as for the photosynthesis genes; however, this enrichment had good statistical support. These classes include histidine metabolism, aminoacyl-tRNA biosynthesis, genes encoding subunits of the NAD $(\mathrm{P}) \mathrm{H}-$ quinone oxidoreductase, ribosomal proteins and terpenoid backbone biosynthesis, which reached enrichment scores between 3.5 and 1.4. Especially for the ribosomal proteins, the number of genes with similar transcript initiation is even higher because many of these genes are organized in huge operons. These data suggest that the gene regulation of many housekeeping processes underlies a conserved regulation.

\section{$5^{\prime}$ UTRs in Prochlorococcus}

The median distance from the gTSS to the start codon of protein-coding genes in Prochlorococcus turned out to be very short, with 26 and 28 nt for MED4 and MIT9313 (Figure 1), respectively, compared with $42 \mathrm{nt}$ in Synechocystis PCC6803 (Mitschke et al., 2011) or $43 \mathrm{nt}$ in $H$. pylori (Sharma et al., 2010). Half of all MED4 (56\%) and MIT9313 (53\%) protein-coding genes have a 5'UTR length between 10 and 40 nt with a maximum frequency at 17 and $15 \mathrm{nt}$, respectively (Figure 1). We searched for the Shine-Dalgarno sequence 'RGGRGG' in all MED4 and MIT9313 5'UTRs excluding those shorter than 17 or $15 \mathrm{nt}$ and allowing one mismatch. Of the 905 MED4 5'UTRs and the 1202 MIT9313 5'UTRs, 29 MED4 genes and 156 MIT9313 genes possess a possible Shine-Dalgarno sequence in the -4 to -23 region. This frequency equals that of a randomly chosen 6-nt-long motif. When searching for the complementary 'YCCYCC' sequence, we detected very similar numbers of 23 MED4 and 161 MIT9313 $5^{\prime}$ UTRs with that motif in the same search region. These findings point to a little if any functional role of a Shine-Dalgarno sequence in Prochlorococcus. In E. coli, the Shine-Dalgarno sequence is bound by the anti-Shine-Dalgarno sequence of the 16S rRNA, anchoring the $30 \mathrm{~S}$ ribosomal subunit around the start codon to form an initiation complex that covers the -35 to +19 region (Hüttenhofer and Noller, 1994). Although the 16S rRNA sequence of Prochlorococcus carries the same anti-SD sequence as described for E. coli, the absence of a Shine-Dalgarno sequence and the high number of protein-coding genes with relatively short $5^{\prime}$ UTRs suggest alternative modes of translation initiation preferentially used in Prochlorococcus. Other mechanisms for translation initiation in E. coli such as the binding of ribosomal protein S1 to the $5^{\prime}$ UTR in mRNAs are also known (Boni et al., 1991) and could be the preferred mechanism for translation initiation used in Prochlorococcus. In many cyanobacteria, two homologous ribosomal S1 proteins Rps1a and Rps1b exist (Figure 2). Rps1a proteins are more closely related to their orthologs than to their paralogous Rps1b protein. Furthermore, orthologous Rps1b proteins seem to be more diverse than orthologous Rps1a proteins. The conservation of duplicated S1 proteins among cyanobacteria indicates an important role of Rps1b; however, whether it is also involved in translation remains enigmatic. The two homologs in MED4 and MIT9313 are predicted to be 40.8 and $45.1 \mathrm{kDa}(34 \%$ sequence identity) and 40.5 and $45.2 \mathrm{kDa}(29 \%$ sequence identity) in size. Mutsuda and Sugiura (Mutsuda and Sugiura, 2006) showed that the $38 \mathrm{kDa} \mathrm{S} 1$ protein (Rps1a) of Synechococcus elongatus PCC 6301 is involved in the efficient initiation of translation via the association with pyrimidine-rich sequences, regardless of the presence or absence of the Shine-Dalgarno sequence. S. elongatus Rps1a exhibits a sequence identity of $75 \%$ to the Prochlorococcus homologs (40.8 $\mathrm{kDa}$ in MED4 and $40.5 \mathrm{kDa}$ in MIT9313), whereas the identity of $S$. elongatus Rps1b within the central-to-C terminal region is only $34 \%$ and $38 \%$, respectively $(45.1 \mathrm{kDa}$ in MED4 and $45.2 \mathrm{kDa}$ in MIT9313).

Another mechanism of translation initiation exists for leaderless mRNAs that lack a $5^{\prime}$ UTR and directly bind a $70 \mathrm{~S}$ ribosome complemented with $\mathrm{N}$-formyl-methionyl-transfer RNA (Moll et al., 2002). Here we found in both strains $\sim 8 \%$ of all gTSS to be located within the first $10 \mathrm{nt}$ to the initiation codon of translation, suggesting that leaderless translation occurs in Prochlorococcus. This is in agreement with computational predictions based on 953 bacterial and 72 archaeal genomes, which suggested that leaderless transcription is widespread among bacteria (207 of the 953 genomes) although not dominant (Zheng et al., 2011). Transcription starts for 30 MED4 ORFs and 41 MIT9313 ORFs on the first nucleotide of the start codon. For 21 MED4 and 34 MIT9313 genes, this is the only detectable TSS under standard growth conditions. Except for three homologs, leaderless transcripts are not conserved between MED4 and MIT9313 and are distributed evenly over the entire genome. The only exceptions are the $\operatorname{ruvB}, \operatorname{deg} T$ and the PMED4_05961/P9313 15281 homologs, encoding the Holliday junction DNA helicase RuvB, a DegT/DnrJ/EryC1/StrS 

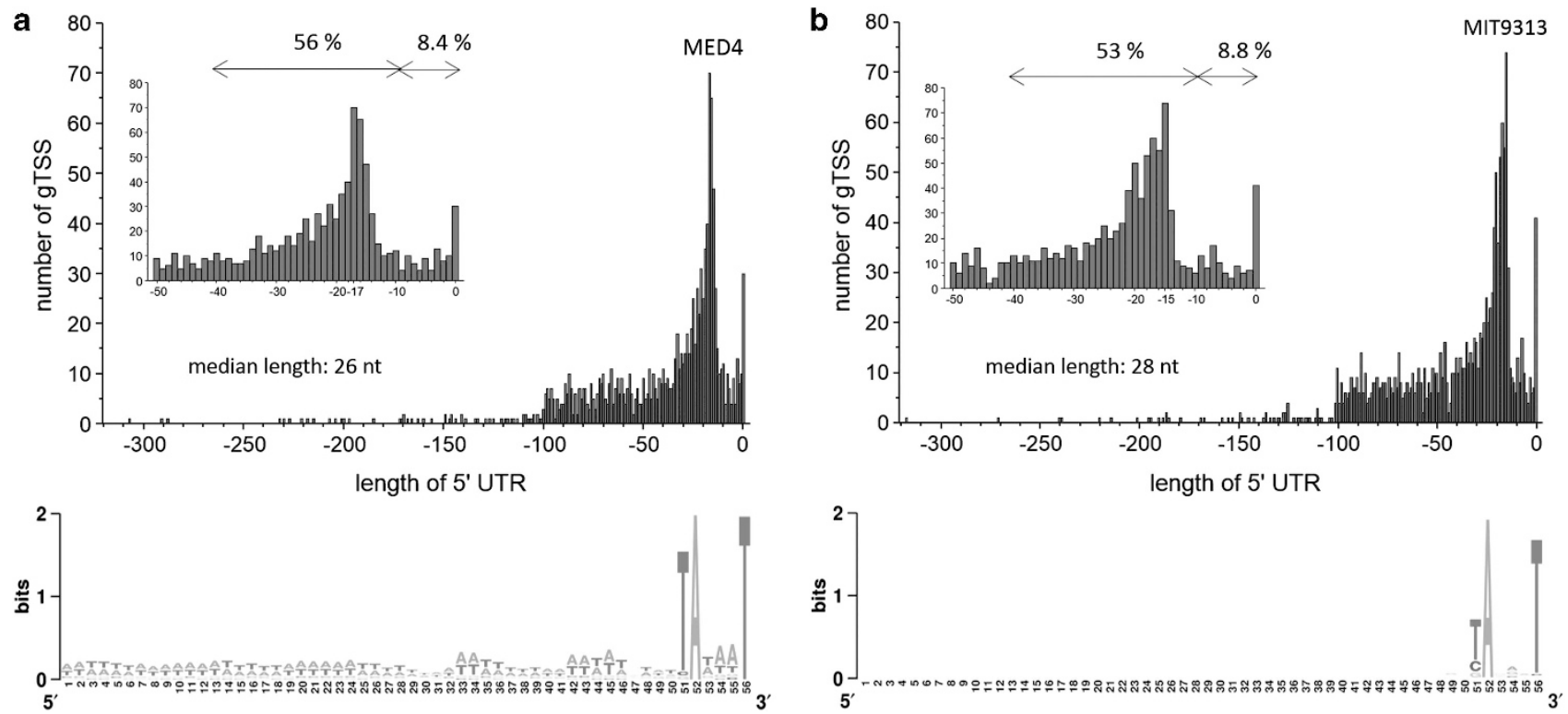

Figure 1 Promoter as well as 5'UTR characteristics of MED4 (a) and MIT9313 (b) gTSS. Based on 1182 MED4 and 1383 MIT9313 gTSS, a minus - 10 element motif 6-8 nt upstream of the transcription initiation site was detected, followed by an upstream periodic AT-stretch signal in MED4. Extremely short 5'UTRs with a median length of 26 nt (MED4) and 28 nt (MIT9313) and the absence of a conserved Shine-Dalgarno sequence suggest alternative modes of action for translation initiation independent of 16S rRNA-mediated ribosome binding. The common 5'UTR length of MED4 is $17 \mathrm{nt}$ and of MIT9313 is $15 \mathrm{nt}$. The majority of protein-coding genes have a 5'UTR length between 10 and $40 \mathrm{nt}$.

aminotransferase family protein and the previously undetected carboxysome shell protein CsoS1d (Klein et al., 2009). All of these three proteins are highly conserved throughout the cyanobacterial phylum; therefore, their leaderless translation might be conserved beyond the two strains studied here. Together, our data indicate that S1 protein-mediated and leaderless translation constitute mechanisms for translation initiation, whereas Shine-Dalgarno sequence-dependent translation initiation plays a secondary role (if at all) in Prochlorococcus. Zheng et al. (2011) determined cyanobacterial-specific sequence signatures in the $5^{\prime} \mathrm{UTR}$ and suggested that other so far unknown mechanisms of translation initiation must exist, which is in total agreement with our results.

\section{Alternative TSS in prochlorosin genes}

Our data show a high number of TSS located within the coding region of protein-coding genes, of which only 14 iTSS (transcripts that initiate within a gene) are conserved among the 377 shared MED4-MIT9313 genes associated with an iTSS. Some of the iTSS are actually gTSS of incorrectly annotated start codons due to automatic ORF calling that usually defines the longest possible ORF as a CDS region. Of the 22 MED4 and 155 MIT9313 iTSS within the first $50 \mathrm{nt}$ of an annotated CDS, which do not possess an additional gTSS, 3 MED4 and 61 MIT9313 iTSS were wrongly classified because of incorrect annotation of the start codon. We have corrected these and reclassified them as gTSS (Supplementary Tables S4 and S5). The iTSS located in the $3^{\prime}$ regions of ORFs that are in the vicinity of a downstream following ORF could give rise to UTRs of the downstream ORF of at least $101 \mathrm{nt}$. However, there is evidence for a subclass of iTSS that gives rise to shorter, very likely functionally relevant transcripts. Among those are TSS that are located within prochlorosin (procA) genes, of which 29 homologs exist in MIT9313 (Li et al., 2010). Prochlorosins are lantipeptides, which are ribosomally translated and posttranslationally modified in the C-terminal core region of the precursor peptide and are distinguishable by the thioether amino acids lanthionine and methyllanthionine (Willey and Van der Donk, 2007). Maturation of the procA is completed by the proteolytic removal of the N-terminal leader sequence, which itself is not modified, resulting in an active metabolite (Willey and Van der Donk, 2007). All 29 homologs are expressed under standard growth conditions, although at a low level of expression (Supplementary Table S10). For 11 of the 29 procA genes, transcription starts exclusively upstream of the annotated gene, whereas 16 proc $A$ homologs possess a gTSS and at least one additional iTSS (Supplementary Table S8). Interestingly, the median distance of the iTSS to the C-terminal core peptide is $26 \mathrm{nt}$, which is very similar to the median $5^{\prime} \mathrm{UTR}$ length of $28 \mathrm{nt}$ that was determined for all MIT9313 gTSS.

Northern hybridizations with specific probes that target either the $5^{\prime} \mathrm{UTR}$ plus the N-terminal region of procA1.4 or the C-terminus including the iTSS gave distinct signals that could be assigned to the fulllength transcripts of the precursor peptide and also to transcripts that start at the mapped iTSS and cover the entire core peptide (Figure 3). The functional 

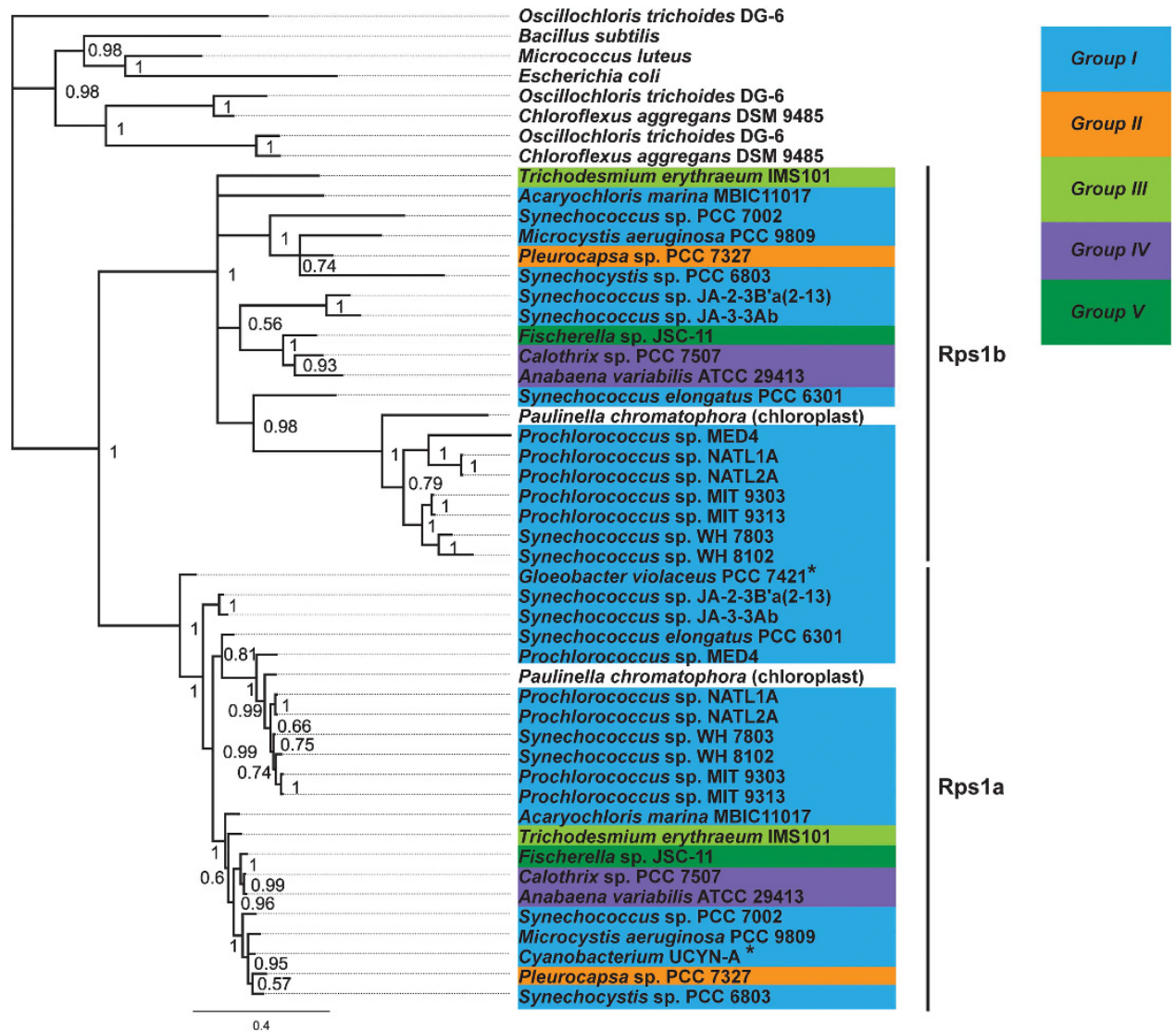

Figure 2 Bayesian inference of phylogeny (Huelsenbeck \& Ronquist 2001) for ribosomal S1 proteins in cyanobacteria, gram-positive bacteria and gram-negative bacteria based on the alignment in Supplementary File S7. We used a poisson rate matrix, gamma distributed rate variation, Oscillochloris trichoides DG-6 as an outgroup and stopped after the average s.d. of split frequencies of 0.016 was reached. Numbers at the nodes represent the posterior probability. The grouping of cyanobacteria is according to Schirrmeister et al., 2011. Cyanobacteria marked with an asterisk (Gloeobacter violaceus PCC 7421 and Cyanobacterium UCYN-A) are devoid of the Rps1b homolog.

relevance of these $3^{\prime}$ transcripts remains enigmatic as the core peptide does not start with a methionine or an alternative start codon. There are two proc $A$ genes-procA2.3 and procA4.1-that are transcribed from an iTSS only. Intriguingly, the core peptide of procA2.3 starts with a methionine and shows distinct signals of 70-, 90-, 200- and 400-nt length when probing against the $3^{\prime} \mathrm{UTR}$ region of the core peptide (Figure 3). The 200-nt fragment is the most abundant of all detected and could encompass the entire core peptide plus a $3^{\prime} U T R$ of 80 nt. In agreement with sequencing data, when probing against the $5^{\prime}$ UTR region, signals were observed neither under standard growth conditions (Figure 3) nor during adaptation to various stress conditions (data not shown). The transcription of only the procA2.3 core peptide raises the question of whether a translated core peptide without the leader peptide could be transformed into a bioactive form at all; the common maturation pathway starts with the posttranslational modification of the precursor peptide by the bifunctional lanthionine synthetase and requires at least certain parts of the leader peptide for substrate binding of the enzyme (Xie et al., 2004), or if the core peptide cannot be modified, thus fulfilling another function. If the prochlorosin could be converted into a bioactive compound by an alternative pathway, a completely new tool set for the synthesis of lantipeptides would become available-a group of small peptides that are already routinely used by the food industry as a preservative and in the clinical field for the eradication of infections caused by multi-drugresistant pathogens (Piper et al., 2009).

High incidence of short cis-encoded asRNAs

The ubiquitous occurrence of cis-encoded asRNAs has been reported for various bacteria (reviewed in Georg and Hess, 2011), and every new transcriptome data set published reveals new examples of antisense transcription. However, we were surprised by the high number of genes that were associated with at least one asRNA. In total, 1117 and 1789 genes with an asRNA within the 454 data set as well as 1600 and 2280 genes within the Solexa data set were determined for MED4 and MIT9313, respectively. The shared number of genes with an asRNA in both the Solexa and 454 data sets was 1008 (54\% of all genes) and 1625 (57\% of all genes) for MED4 and MIT9313, respectively (Figure 3). We furthermore applied an alternative approach to the differential RNA-seq method and hybridized Prochlorococcus-specific 
a

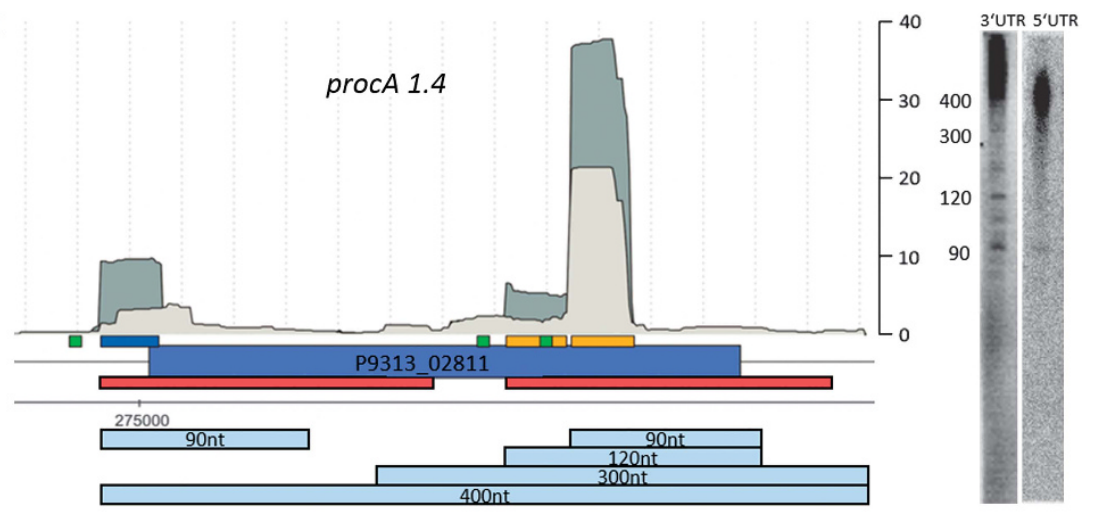

b

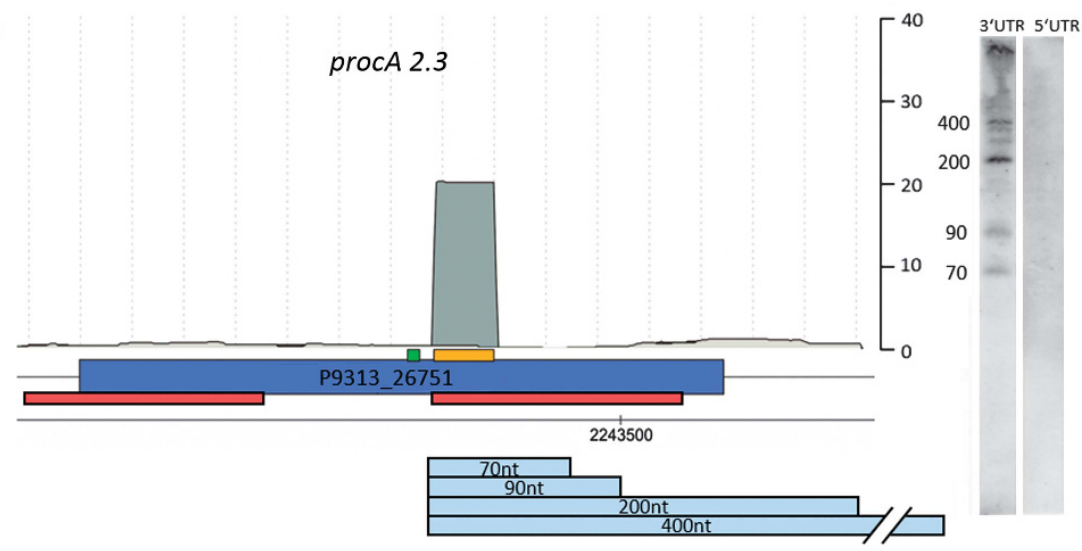

Figure 3 Overview on procA genes procA1.4 (a) and procA2.3 (b) read distribution based on mapped Solexa reads of the [ + ] $($ dark gray lines) and [ - ] (light gray lines) cDNA libraries and northern blot hybridizations with probes (red boxes) recognizing either the $5^{\prime}$ region or the $3^{\prime}$ region. Regions of mapped reads that give rise to a TSS are marked with blue (gTSS) and orange (iTSS) boxes. The - 10 element is marked with a green box. Transcript lengths as determined by northern hybridization are given as light blue boxes. A total of $10 \mu \mathrm{g}$ of total RNA was loaded on $10 \%$ PAA gels and blotted onto nitrocellulose membranes.

Affymetrix microarrays with directly Cy3-labeled RNA from cultures grown under standard conditions or several stress conditions (for more details see Material and Methods) (Supplementary Files S8 and S9). Because probes of these microarrays were initially designed for hybridization with cDNA, signal intensities above the threshold would provide information on the existence and expression of asRNAs when hybridizing with directly labeled RNA. In total, we found 1875 and 2613 genes with an associated asRNA for MED4 and MIT9313, respectively, confirming a potentially global antisense transcription in Prochlorococcus. A high degree of antisense transcription has been previously reported for MED4 (Waldbauer et al., 2012) and appears to be a common feature not only for high-light-adapted ecotypes but also for low-lightadapted strains according to the presented data. Notably, the overlap between the three methods used for the identification of asRNAs was smaller than expected, which can be explained by distinct biases of the different technologies (Harismendy et al., 2009; Raabe et al., 2014). To ensure the validity of our analysis, we considered only those asRNAs that were confirmed by at least two methods (Supplementary Figure S1). As a result, for both
MED4 and MIT9313, approximately three-quarters of all genes possess an asRNA.

To obtain a better understanding of the characteristics of asRNAs in Prochlorococcus, we determined the average transcript length and the relative abundance of asRNAs in comparison with mRNAs. For that analysis, aTSS and gTSS reads were assembled to longer transcripts using the segmentation algorithm of RNASeg (to be published elsewhere). The stoichiometric mean ratio of mRNA to asRNA reads was 19 and 15, and the average asRNA length was 242 and $408 \mathrm{nt}$ for MIT9313 and MED4, respectively. Of 616 shared genes with an associated asRNA, only 52 asRNAs were conserved with respect to position. Because we noticed a very dense promoter occurrence in general for both MIT9313 (every $353 \mathrm{nt}$ ) and MED4 (every $563 \mathrm{nt}$ ), these short asRNAs could solely be stable non-functional by-products - for example, in the form of doublestranded RNA.

However, the sheer abundance of asRNAs spurred us to investigate the functional relevance of this class of molecules in more detail. We chose putative asRNAs of MED4 and MIT9313 and tested their possible differential expression under various stress conditions. All tested asRNAs showed an altered 
expression behavior in one or more conditions compared with standard growth conditions (Figure 4). CynS (PMM0373) encodes a cyanase that converts cyanate to carbon dioxide and ammonia and appears to have a role in cyanate utilization rather than in detoxification in MED4 (Kamennaya and Post, 2011). Intriguingly, under nitrogen-limiting conditions, an asRNA of $\sim 300 \mathrm{nt}$ is induced that may have an important role in the regulation of cynS (Figure 4a). YocE (PMM1378, desA) encodes a fatty acid desaturase, type II, and its expression is induced during low temperature and high light in Synechococcus PCC7002 (Sakamoto et al., 1997, 1998). Our data suggest a potential yocE asRNAmediated regulation of yocE during stationary phase and iron-limiting conditions (Figure 4a). The mRNA of the manganese-binding photosystem II protein Y, encoded by $p s b Y$, is covered by a 120-nt-long asRNA (Figure 4b). PsbY and the gene immediately downstream, gidA (PMT1049, containing a NAD-binding domain), appear to be organized in a dicistron (Figure 4b), and the asRNA of psbY may enable the decoupling of the gene expression regulation of $p s b Y$ and gidA. An $\sim 130$-nt asRNA covers the $3^{\prime}$ region of P9313_10131—a gene with homology to the thiol oxidoreductase of Synechococcus CC9311 (Figure 4b). The specific decrease in the asRNA abundance under nitrogen starvation suggests its involvement in the gene expression regulation of P9313_10131.

These few examples point to a very complex regulatory network through asRNAs and emphasize the important role of functional RNAs in the gene regulation of Prochlorococcus.

\section{Non-coding RNAs are light clade-specific in Prochlorococcus}

Gene expression control through ncRNAs constitutes an important regulatory pathway in MED4 (Axmann et al., 2005; Steglich et al., 2008). However, until now, there has been little information on the ncRNA content in other Prochlorococcus strains. Of the 20 previously described ncRNAs in MED4 (Axmann et al., 2005; Steglich et al., 2008), 16 were also detected here using the differential RNA-seq approach approach. Additionally, we found six new
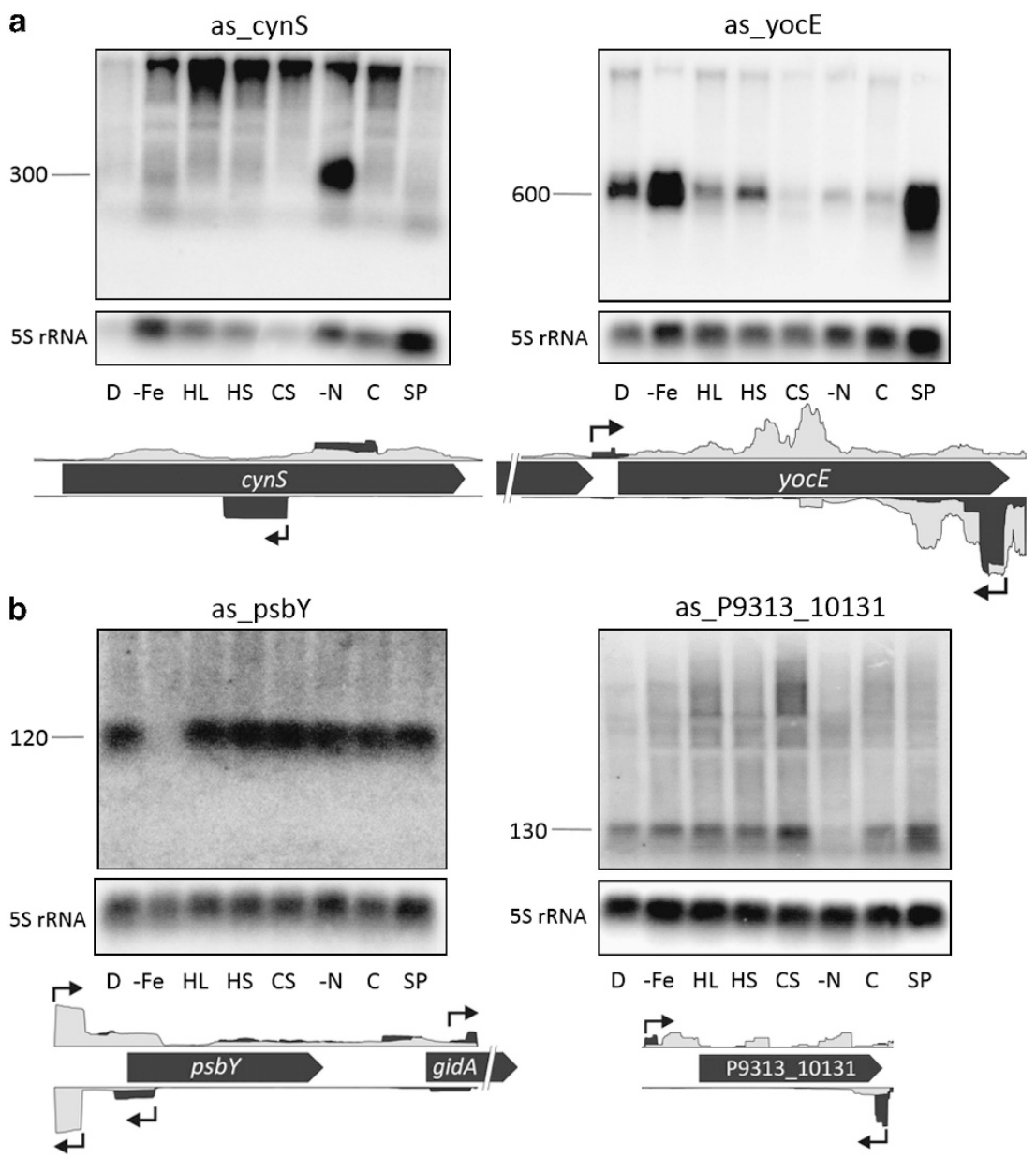

Figure 4 Verification of selected asRNAs of MED4 (a) and MIT9313 (b), and determination of expressional changes during several stress conditions (D—darkness, -Fe—iron depletion, HL—high light, HS—heat shock, CS—cold shock, -N—nitrogen deprivation, C-standard growth conditions and SP-stationary phase) by northern blots. 5S RNA was used as a loading control. The gene arrangement and coverage with reads of the primary (black) or secondary (gray) library are shown at the bottom. Arrows indicate positions of TSS. 

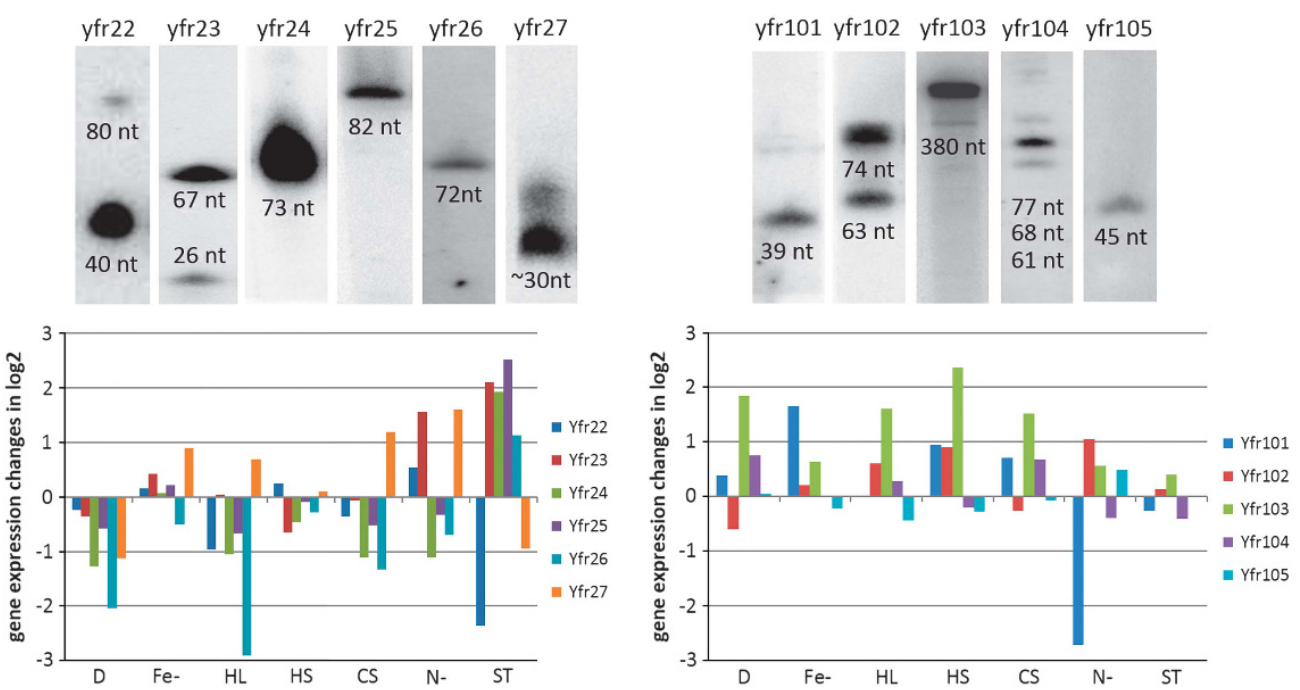

Figure 5 Verification of ncRNA candidates by northern blot hybridization. Different stress conditions have been applied for MED4 and MIT9313 and $5 \mu$ g of extracted total RNA was separated on PAA gels and blotted onto nitrocellulose membranes. The size of RNAs was estimated by RNA ladder interpolation and from sequencing data. Fold changes (normalized against the internal standard 5S rRNA) of seven different stresses in comparison with the control hybridization are given in log2.

ncRNAs either through manual inspection of nTSS or via computational prediction using RANDfold (Bonnet et al., 2004) (Figure 5 and Supplementary Figure S2 and Supplementary Table S9). For MIT9313, only five ncRNAs that are all homologs of MED4 ncRNAs have been previously identified. Applying the same search criteria as for MED4, we detected five additional ncRNAs in MIT9313 and some potential ncRNA candidates, however with ambiguous hybridization results (Supplementary Figure S3). From sequencing results (300 nTSS in the 454 data set and 639 in the Solexa data) we can expect more ncRNAs to be detected in MIT9313. The five new identified ncRNAs in MIT9313 only occur (in contrast to the known ncRNAs) in lowlight-adapted ecotypes (Figure 5 and Supplementary Figure S4 and Supplementary Table S10). The only exception is Yfr103, which also exists in Synechococcus strains CC9311, CC9605 and CC9902. In the same manner, homologs of the newly detected MED4 ncRNAs are restricted to the high-light clade or exclusively occur in MED4-for example, Yfr26 and Yfr27. From this, we conclude that most ncRNAs of Prochlorococcus are high-light- and low-light-clade specific, which indicates the functional importance of these regulators for the adaptation to clade-specific niches. It is well documented that ncRNAs are frequently coregulated with the environmental conditions in which they have a role. Therefore, it is consistent that the gross of tested ncRNAs responded during the adaptation to light, nutrient and temperature fluctuations (Figure 5). The strongest response of ncRNA expression was detectable during nitrogen deprivation and the stationary phase. The adaptation to nitrogen-limiting conditions and stationary phase through regulatory circuits involving ncRNAs has been previously reported for other bacteria (Jäger et al., 2009; Fröhlich et al., 2012).

In summary, our data support the previously suggested importance of ncRNAs in the regulatory network of MED4, a view that now can be expanded to the low-light-adapted strain MIT9313 and probably Prochlorococcus in general.

\section{Conclusions and possible implications}

We discovered a dense promoter activity in both strains and very diverse transcriptome architectures between MED4 and MIT9313. A major fraction of newly identified transcripts is represented by asRNAs, which infers that the transcript pool is nearly twice as complex as the annotated gene content in both MED4 and MIT9313. The accumulation of short asRNAs may be a factor contributing to the reported short global RNA half-life times (2.4 minutes) (Steglich et al., 2010) despite Prochlorococcus' slow doubling times of usually one per day (Partensky et al., 1999). Not only are these short asRNAs upon pairing to mRNA perfect substrates for ribonuclease III, which recognizes double-stranded RNA, but the partial overlap of mRNA - asRNA duplexes could also generate additional entry sites in single-stranded regions for ribonuclease E (Stazic et al., 2011), which is the major ribonuclease in regulated mRNA ribolysis. Thus, a high substrate availability for ribonuclease might lead to enhanced RNA turnover rates. The high number of asRNAs compared protein regulators in Prochlorococcus could explain the short length of the $5^{\prime}$ UTRs; these regions are essential for gene regulation through protein regulators (and trans-acting ncRNAs) but are less important for asRNA-mediated gene regulation. 


\section{Conflict of Interest}

The authors declare no conflict of interest.

\section{Acknowledgements}

We thank Stephanie Hein for providing RNA of stressed MED4 cultures for microarray analyses and Jörg Vogel for helping with 454 sequencing. The research was supported by the DFG (SPP 1258) to CS and WRH and the EU project MaCuMBA (grant agreement no: 311975 ) to WRH.

\section{References}

Axmann IM, Kensche P, Vogel J, Kohl S, Hess WR. (2005). Identification of cyanobacterial non-coding RNAs by comparative genome analysis. Genome Biol 6: R73.

Boni IV, Isaeva DM, Musychenko ML, Tzareva NV. (1991). Ribosome-messenger recognition: mRNA target sites for ribosomal protein S1. Nucleic Acids Res. 19: $155-162$.

Bonnet E, Wuyts J, Rouzé P, Van de Peer Y. (2004). Evidence that microRNA precursors, unlike other noncoding RNAs, have lower folding free energies than random sequences. Bioinformatics 20: 2911-2917.

Dufresne A, Salanoubat M, Partensky F, Artiguenave F, Axmann IM, Barbe V et al. (2003). Genome sequence of the cyanobacterium Prochlorococcus marinus SS120, a nearly minimal oxyphototrophic genome. Proc Natl Acad Sci USA 100: 10020-10025.

Fröhlich KS, Papenfort K, Berger AA, Vogel J. (2012). A conserved RpoS-dependent small RNA controls the synthesis of major porin OmpD. Nucleic Acids Res 40: 3623-3640.

Garczarek L, Partensky F, Irlbacher H, Holtzendorff J, Babin M, Mary I et al. (2001). Differential expression of antenna and core genes in Prochlorococcus PCC 9511 (Oxyphotobacteria) grown under a modulated light-dark cycle. Environ Microbiol 3: 168-175.

Georg J, Voss B, Scholz I, Mitschke J, Wilde A, Hess WR. (2009). Evidence for a major role of antisense RNAs in cyanobacterial gene regulation. Mol Syst Biol 5: 1-17.

Georg J, Hess WR. (2011). cis-antisense RNA, another level of gene regulation in bacteria. Microbiol. Mol Biol Rev 75: $286-300$.

Goericke R, Welschmeyer NA. (1993). The marine prochlorophyte Prochlorococcus contributes significantly to phytoplankton biomass and primary production in the Sargasso Sea. Deep Sea Res 40: 2283-2294.

Grote J, Thrash JC, Huggett MJ, Landry ZC, Carini P, Giovannoni SJ et al. (2012). Streamlining and core genome conservation among highly divergent members of the SAR11 clade. MBio 3: pii: e00252-12.

Harismendy O, Ng PC, Strausberg RL, Wang X, Stockwell TB, Beeson KY et al. (2009). Evaluation of next generation sequencing platforms for population targeted sequencing studies. Genome Biol. 10: R32.

Hoffmann S, Otto C, Kurtz S, Sharma C, Khaitovich P, Vogel J et al. (2009). Fast Mapping of Short Sequences with Mismatches, Insertions and Deletions Using Index Structures. PLoS Comput Biol 5: e1000502.

Huelsenbeck JP, Ronquist F. (2001). MRBAYES: Bayesian inference of phylogenetic trees. Bioinformatics 17: $754-755$.
Hüttenhofer A, Noller HF. (1994). Footprinting mRNAribosome complexes with chemical probes. EMBO J 13: 3892-3901.

Jäger D, Sharma CM, Thomsen J, Ehlers C, Vogel J, Schmitz RA. (2009). Deep sequencing analysis of the Methanosarcina mazei Gö1 transcriptome in response to nitrogen availability. Proc Natl Acad Sci USA 106: 21878-21882.

Johnson ZI, Zinser ER, Coe A, McNulty NP, Woodward EMS, Chisholm SW. (2006). Niche partitioning among Prochlorococcus ecotypes along ocean-scale environmental gradients. Science 311: 1737-1740.

Kamennaya NA, Post AF. (2011). Characterization of cyanate metabolism in marine Synechococcus and Prochlorococcus spp. Appl Environ Microbiol 77: 291-301.

Kettler GC, Martiny AC, Huang K, Zucker J, Coleman ML, Rodrigue S et al. (2007). Patterns and implications of gene gain and loss in the evolution of Prochlorococcus. PLoS Genet 3: e231.

Klein MG, Zwart P, Bagby SC, Cai F, Chisholm SW, Heinhorst $S$ et al. (2009). Identification and structural analysis of a novel carboxysome shell protein with implications for metabolite transport. J Mol Biol 392: 319-333.

Li B, Sher D, Kelly L, Shi Y, Huang K, Knerr PJ et al. (2010). Catalytic promiscuity in the biosynthesis of cyclic peptide secondary metabolites in planktonic marine cyanobacteria. Proc Natl Acad Sci USA 107: 10430-10435.

Li S, Xu M, Su Z. (2010). Computational analysis of LexA regulons in Cyanobacteria. BMC Genomics 11: 527.

Lindell D, Jaffe JD, Coleman ML, Futschik ME, Axmann IM, Rector T et al. (2007). Genome-wide expression dynamics of a marine virus and host reveal features of co-evolution. Nature 449: 83-86.

Martiny AC, Coleman ML, Chisholm SW. (2006). Phosphate acquisition genes in Prochlorococcus ecotypes: evidence for genome-wide adaptation. Proc Natl Acad Sci USA 103: 12552-12557.

Mitschke J, Georg J, Scholz I, Sharma CM, Dienst D, Bantscheff J et al. (2011). An experimentally anchored map of transcriptional start sites in the model cyanobacterium Synechocystis sp. PCC6803. Proc Natl Acad Sci USA 108: 2124-2129.

Moll I, Grill S, Gualerzi CO, Bläsi U. (2002). Leaderless mRNAs in bacteria: surprises in ribosomal recruitment and translational control. Mol Microbiol 43: 239-246.

Moore LR, Coe A, Zinser ER, Saito MA, Sullivan MB, Lindell D et al. (2007). Culturing the marine cyanobacterium Prochlorococcus. Limnol Oceanogr Meth 5: 353-362.

Moore LR, Goericke R, Chisholm SW. (1995). Comparative physiology of Synechococcus and Prochlorococcus: influence of light and temperature on growth, pigments, fluorescence and absorptive properties. Mar Ecol Prog Ser 116: 259-275.

Mutsuda M, Sugiura M. (2006). Translation initiation of cyanobacterial rbcS mRNAs requires the $38-\mathrm{kDa}$ ribosomal protein $\mathrm{S} 1$ but not the Shine-Dalgarno sequence: development of a cyanobacterial in vitro translation system. J Biol Chem 281: 38314-38321.

Partensky F, Hess WR, Vaulot D. (1999). Prochlorococcus, a marine photosynthetic prokaryote of global significance. Microbiol Mol Biol Rev 63: 106-127.

Pinto FL, Thapper A, Sontheim W, Lindblad P. (2009). Analysis of current and alternative phenol based RNA extraction methodologies for cyanobacteria. BMC Mol Biol 10: 79. 
Piper C, Cotter P, Ross R, Hill C. (2009). Discovery of medically significant lantibiotics. Curr Drug Discovery Technol 6: 1-18.

Raabe CA, Tang T-H, Brosius J, Rozhdestvensky TS. (2014). Biases in small RNA deep sequencing data. Nucleic Acids Res 42: 1414-1426.

Richter AS, Schleberger C, Backofen R, Steglich C. (2010). Seed-based INTARNA prediction combined with GFP-reporter system identifies mRNA targets of the small RNA Yfr1. Bioinformatics 26: 1-5.

Rocap G, Larimer FW, Lamerdin J, Malfatti S, Chain P, Ahlgren NA et al. (2003). Genome divergence in two Prochlorococcus ecotypes reflects Oceanic niche differentiation. Nature 424: 1042-1047.

Sakamoto T, Higashi S, Wada H, Murata N, Bryant DA. (1997). Low-temperature-induced desaturation of fatty acids and expression of desaturase genes in the cyanobacterium Synechococcus sp. PCC 7002. FEMS Microbiol Lett 152: 313-320.

Sakamoto T, Shen G, Higashi S, Murata N, Bryant DA. (1998). Alteration of low-temperature susceptibility of the cyanobacterium Synechococcus sp. PCC 7002 by genetic manipulation of membrane lipid unsaturation. Arch Microbiol 169: 20-28.

Scanlan DJ, Ostrowski M, Mazard S, Dufresne A, Garczarek L, Hess WR et al. (2009). Ecological genomics of marine picocyanobacteria. Microbiol Mol Biol Rev 73: 249-299.

Schirrmeister BE, Antonelli A, Bagheri HC. (2011). The origin of multicellularity in cyanobacteria. $B M C$ Evol Biol 11: 45.

Sharma CM, Hoffmann S, Darfeuille F, Reignier J, Findeiss S, Sittka A et al. (2010). The primary transcriptome of the major human pathogen Helicobacter pylori. Nature 464: 250-255.

Sittka A, Lucchini S, Papenfort K, Sharma CM, Rolle K, Binnewies TT et al. (2008). Deep sequencing analysis of small noncoding RNA and mRNA targets of the global post-transcriptional regulator, Hfq. PLoS Genet 4: e1000163.

Smyth G. (2005). limma: Linear models for microarray data. In Gentleman R, Carey VJ, Huber W, Irizarry RA, Dudoit S (eds Bioinformatics and Computational Biology Solutions Using $R$ and Bioconductor. Statistics for Biology and Health, Springer: New York, NY, USA, pp. 397-420.

Stazic D, Lindell D, Steglich C. (2011). Antisense RNA protects mRNA from RNase E degradation by RNARNA duplex formation during phage infection. Nucleic Acids Res 39: 4890-4899.

Steglich C, Frankenberg-Dinkel N, Penno S, Hess WR. (2005). A green light-absorbing phycoerythrin is present in the high-light-adapted marine cyanobacterium Prochlorococcus sp. MED4. Environ Microbiol 7: 1611-1618.

Steglich C, Futschik M, Rector T, Steen R, Chisholm SW. (2006). Genome-wide analysis of light sensing in Prochlorococcus. J Bacteriol 188: 7796-7806.
Steglich C, Futschik ME, Lindell D, Voss B, Chisholm SW, Hess WR. (2008). The challenge of regulation in a minimal phototroph: Non-coding RNAs in Prochlorococcus. PLoS Genet 4: e10000173.

Steglich C, Lindell D, Futschik M, Rector T, Steen R, Chisholm SW. (2010). Short RNA half-lives in the slow-growing marine cyanobacterium Prochlorococcus. Genome Biol 11: R54.

Su Z, Olman V, Xu Y. (2007). Computational prediction of Pho regulons in cyanobacteria. BMC Genomics 8: 156.

Swan BK, Tupper B, Sczyrba A, Lauro FM, Martinez-Garcia M, González JM et al. (2013). Prevalent genome streamlining and latitudinal divergence of planktonic bacteria in the surface ocean. Proc Natl Acad Sci USA 110: 11463-11468.

Thompson AW, Huang K, Saito MA, Chisholm SW. (2011). Transcriptome response of high- and low-lightadapted Prochlorococcus strains to changing iron availability. ISME J 5: 1580-1594.

Thompson LR, Field C, Romanuk T, Ngugi D, Siam R, El Dorry $\mathrm{H}$ et al. (2013). Patterns of ecological specialization among microbial populations in the Red Sea and diverse oligotrophic marine environments. Ecol Evol 3: 1780-1797.

Tolonen AC, Aach J, Lindell D, Johnson ZI, Rector T, Steen R et al. (2006). Global gene expression of Prochlorococcus ecotypes in response to changes in nitrogen availability. Mol Syst Biol 2: 53.

Vaulot D, Marie D, Olson RJ, Chisholm SW. (1995). Growth of Prochlorococcus, a photosynthetic prokaryote, in the equatorial Pacific Ocean. Science 268: 1480-1482.

Vogel J, Axmann I, Herzel H, Hess WR. (2003). Experimental and computational analysis of transcriptional start sites in the cyanobacterium Prochlorococcus MED4. Nucleic Acids Res 31: 2890-2899.

Waldbauer JR, Rodrigue S, Coleman ML, Chisholm SW. (2012). Transcriptome and proteome dynamics of a light-dark synchronized bacterial cell cycle. PLoS One 7: e43432.

Willey JM, Van der Donk WA. (2007). Lantibiotics: Peptides of diverse structure and function. Ann Rev Microbiol 61: 477-501.

Xie L, Miller LM, Chatterjee C, Averin O, Kelleher NL, van der Donk WA. (2004). Lacticin 481: In Vitro reconstitution of lantibiotic synthetase activity. Science 303: 679-681.

$\mathrm{Xu} \mathrm{M}$, Su Z. (2009). Computational prediction of cAMP receptor protein (CRP) binding sites in cyanobacterial genomes. BMC Genomics 10: 23.

Zheng X, Hu G-Q, She Z-S, Zhu H. (2011). Leaderless genes in bacteria: clue to the evolution of translation initiation mechanisms in prokaryotes. BMC Genomics 12: 361 .

Supplementary Information accompanies this paper on The ISME Journal website (http://www.nature.com/ismej) 